\title{
Programa de Atividade Física Comunitária: tempo necessário e execução
}

\section{Community Physical Activity Program: time requirements and delivery}

\section{Programa de Actividad Física Comunitaria: tiempo necesario y ejecución}

Recebido: 10/06/2013

Aprovado: 21/02/2014

\author{
Samantha Marie Harden 1 \\ Fabio Araújo Almeida ${ }^{2}$ \\ Wendy You ${ }^{3}$ \\ Paul Andrews Estabrooks ${ }^{4}$
}

A dimensão Implementação do RE-AIM avalia a fidelidade ao protocolo de tratamento e seus custos relacionados. Neste estudo, um programa comunitário de atividade física e consumo de frutas e vegetais baseado em evidências foi implementado por educadores em saúde no âmbito do Sistema de Extensão Cooperativa. O objetivo deste estudo foi determinar: 1) a medida em que os educadores em saúde aderiram aos princípios subjacentes e 2) o tempo necessário para planejar, recrutar e executar a intervenção. Dezesseis educadores em saúde $(100,0 \%)$ que aplicaram o programa completaram um questionário que incluiu itens sobre os requisitos de tempo e protocolo de tratamento. Verificou-se que os educadores em saúde aderiam aos três princípios subjacentes do programa, como observado através da criação de grupos $(100,0 \%)$, coleta do alcance de metas dos grupos $(80,1 \%, \mathrm{SD} \pm 0,08 \%)$, e fornecimento de feedback (72,5\%, SD $\pm 0,16 \%)$ ao longo de 8 semanas do estudo. No entanto, quando o programa se aproximou do final (semanas 6-8), houve uma redução significativa $(\mathrm{p}<0,05)$ no fornecimento de feedback para as equipes. Os educadores em saúde passaram mais tempo na coleta de quilômetros percorridos por cada equipe $(12,4 \mathrm{SD} \pm 18,8$ horas), na coleta de informações dos participantes $(9,47 \mathrm{SD} \pm 19,8$ horas), se comunicando com os participantes $(9,32 \mathrm{SD} \pm 13,7$ horas) e no desenvolvimento de materiais locais para boletins informativos $(5,7 \mathrm{SD} \pm 3,79$ horas $)$. Os componentes do programa que os educadores em saúde passaram a menor quantidade de tempo foram na preparação dos pacotes para os lideres de cada equipe $(0,909 \mathrm{SD} \pm 2,7$ horas $)$, na condução de atividades $(2,54 \mathrm{SD} \pm 7,17$ horas $)$ e no acompanhamento dos lideres $(3,09 \mathrm{SD} \pm 2,81$ horas). Este estudo indica que o tempo necessário para o programa Fit Extension se adequou às expectativas dos educadores em saúde. Pesquisas futuras são necessárias para comparar a fidelidade da implementação e estruturas que possam promover a sustentabilidade do programa.

Descritores: Exercício. Relações Comunidade-Instituição. Promoção da saúde. Educadores em saúde. Avaliação de Programas e Projetos de Saúde.

\footnotetext{
1 Bióloga. Doutora em Nutrição Humana, Alimentação e Exercício. Departamento de Cinesiologia. Universidade de British Columbia, Canadá.

2 Assistente Social. Especialista em Práticas Comunitárias. Mestre em Serviço Social com ênfase em Prática Comunitária e Desenvolvimento Internacional. Doutor em Serviço Social. Departamento de Nutrição Humana, Alimentação e Exercício. Universidade Virginia Tech., Estados Unidos da América.

${ }^{3}$ Bacharel em Artes. Especialista em Comércio Internacional. Doutora em Economia Agrária. Departamento de Agricultura e Economia Aplicada. Universidade Virginia Tech., Estados Unidos da América.

4 Educador Físico. Mestre em Cinesiologia. Doutor em Cinesiologia. Departamento de Nutrição Humana, Alimentação e Exercício. Universidade Virginia Tech., Estados Unidos da América. estabrkp@vt.edu.
} 
The implementation dimension of RE-AIM evaluates the fidelity to treatment protocol and its related costs. In this study, an evidence-based, community-wide physical activity and fruit/vegetable consumption program was implemented by health educators within the Cooperative Extension System. The purpose of this study was to determine 1) the extent to which health educators adhere to underlying program principles and 2) the time required to plan, recruit, and deliver the intervention. Sixteen agents (100.0\%) who delivered the program completed a survey that included items on both time requirements and treatment protocol. When analyzed, it was found that health educators adhered to the three underlying principles of the program; as seen by delivering to groups $(100.0 \%)$, collecting group goal attainment $(80.1 \%, \mathrm{SD} \pm .08 \%)$, and providing feedback $(72.5 \%, \mathrm{SD} \pm .16 \%)$ over the 8 week study. However, as the program reached the end (weeks 6-8), there was a significant $(p<.05)$ decline in providing feedback to teams. The health educators spent the most time on collecting team miles (12.4 SD \pm 18.8 hours), entering participant information (9.47 SD \pm 19.8 hours), communicating with participants (9.32 SD \pm 13.7 hours), and developing local materials for newsletters (5.7 SD \pm 3.79 hours). The program components that health educators spent the least amount of time on were preparing captains packets (.909 SD \pm 2.7 hours), leading activities (2.54 SD \pm 7.17 hours), and following up with captains (3.09 SD \pm 2.81 hours). This study indicates that the time requirements for Fit Extension fit well within the expectations of health educators. Future research is needed to compare implementation fidelity and structures that may promote program sustainability.

Descriptors: Exercise; Community-institutional relations; Health promotion; Health educators; Program evaluation.

La dimensión Implementación del RE-AIM evalúa la fidelidad al protocolo de tratamiento y sus costos relacionados. En este estudio, un programa comunitario de actividad física y consumo de frutas y vegetales basado en evidencias fue implementado por educadores en salud en el ámbito del Sistema de Extensión Cooperativa. El objetivo de éste estudio fue determinar: 1) la medida en que los educadores en salud adhirieron a los principios subyacentes y 2) el tiempo necesario para planear, reclutar y ejecutar la intervención. Dieciséis educadores en salud (100\%) que aplicaron el programa, completaron un cuestionario que incluyó ítems sobre los requisitos del tiempo y protocolo de tratamiento. Se verificó que los educadores en salud se adherían a los tres principios subyacentes del programa, como observado a través de la creación de grupos (100\%), colecta del alcance de metas de los grupos $(80,1 \% \mathrm{SD} \pm 0,08 \%)$, y suministro de feedback $(72,5 \%, \mathrm{SD} \pm 0,16 \%)$ a lo largo de 8 semanas de estudio. Sin embargo, cuando el programa se aproximó al final (semanas 6-8), hubo una reducción significativa $(\mathrm{p}<0,05)$ en el suministro de feedback para los equipos. Los educadores en salud pasaron mas tiempo en la colecta de kilómetros recorridos por cada equipo $(12,4 \mathrm{SD} \pm 18,8$ horas) en la colecta de informaciones de los participantes $(9,47 \mathrm{SD} \pm 19,8$ horas $)$,comunicándose con los participantes $(9,32 \mathrm{SD} \pm 13,7$ horas) y en el desenvolvimiento de materiales locales para boletines informativos $(5,7$ $\mathrm{SD} \pm 3,79$ horas). Los componentes del programa que los educadores en salud pasaron en menor cantidad de tiempo fue en la preparación de los paquetes para los líderes de cada equipo $(0,909 \mathrm{SD} \pm 2,7$ horas), en la conducción de actividades $(2,54 \mathrm{SD} \pm 7,17$ horas $)$ y en el acompañamiento de los líderes (3,09 SD+2,81£oras). Este estudio indica que el tiempo para el programa Fit Extension se adecuó a las expectativas de los educadores en salud. Serán necesarias investigaciones futuras para comparar la fidelidad de la implementación y estructuras que puedan promover la sustentabilidad del programa.

Descriptores: Ejercicio; Relaciones Comunidad-Institución; Promoción de la salud; Educadores en salud; Evaluación de Programas y Proyectos de Salud. 


\section{INTRODUÇÃO}

A implementação pode ser definida como o grau em que uma intervenção é executada como pretendido e os seus custos relacionados ${ }^{1}$. Ambas as áreas são importantes na translação de pesquisa para práticas clínicas e comunitárias. Acredita-se que intervenções que são entregues com baixa fidelidade ao protocolo são menos propensas a conseguir um efeito semelhante como foi encontrado em ambientes controlados de pesquisa ${ }^{2}$.

Por sua vez, é evidente que a adaptação ocorre quando os profissionais da prática usam programas baseados em pesquisa $^{3}$. Além disso, as intervenções que têm custos elevados são menos prováveis de serem adaptadas da pesquisa para a prática. Apesar da importância da fidelidade e do custo, há pouca informação sobre esta dimensão do RE-AIM na literatura existente ${ }^{4}$. Este trabalho apresenta a avaliação da implementação de um programa de promoção de atividade física e consumo de frutas e vegetais na comunidade, o Fit Extension, usando o modelo RE-AIM para as avaliações.

Em 2008, a Virginia Cooperative Extension (VCE) identificou a necessidade de abordar a baixa prevalência de atividade física e consumo de frutas e verduras, e se engajou em uma abordagem baseada em sistemas para desenvolver o Fit Extension ${ }^{5,6}$. 0 processo usado para desenvolver 0 programa incluiu o envolvimento dos líderes responsáveis pelas decisões organizacionais, administradores regionais, representantes e educadores em saúde que seriam responsáveis pela execução do programa. Além disso, pesquisadores com experiência em nutrição, atividade física, avaliação e formatação de programas foram incluídos como parte da equipe de desenvolvimento.

A abordagem baseada na Teoria de Sistemas foi utilizada para garantir que o Fit Extension fosse projetado com base em provas científicas sólidas e com a intenção de alinhar-se com a estrutura, recursos e missão do VCE. O programa resultante foi baseado na Teoria de Dinâmica de Grupo ${ }^{7}$ e membros da comunidade foram incentivados a participar como equipes que juntas caminhavam, figurativamente, toda a extensão do Estado da Virgínia durante um período de oito semanas.

As distâncias da atividade física foram calculadas baseadas em 15 minutos de atividade física moderada (30 minutos de bicicleta - 3,22 Km) ou em quilometragem real a $6,76 \mathrm{Km} / \mathrm{h}$ ou mais de velocidade. Além de acompanhar a atividade física, os participantes foram convidados a acompanharem a sua ingestão de frutas e vegetais, com um objetivo global de consumo de cinco xícaras de frutas e vegetais por dia.

Equipes de seis pessoas cada foram selecionadas para alinhar a meta da equipe com as diretrizes recomendadas de cerca de 150 minutos de atividade física moderada para cada membro da equipe por semana. No decorrer do programa, boletins com informações baseadas em evidências eram enviados semanalmente, contendo na última página o feedback local adaptado do programa. Os componentes da intervenção incluíam o objetivo do grupo e, uma vez por semana, os participantes relatavam a atividade física e o recebimento dos boletins informativos com as orientações adaptadas e o feedback da equipe, assim como mensagens baseadas em teorias para promover a atividade física e consumo frutas e vegetais.

Educadores em saúde também foram providos com materiais e informações necessárias para envolver as partes interessadas da comunidade em atividades de planejamento e de recrutamento dos participantes que não estavam diretamente relacionados com a execução do programa em si.

O objetivo deste estudo foi identificar o grau em que os educadores em saúde utilizaram uma abordagem baseada em equipes, com boletins semanais, orientações personalizadas e feedback para as equipes. Um objetivo secundário do estudo foi determinar o tempo necessário para execução do Fit Extension. Este propósito foi subdividido entre tempo gasto na execução direta do programa e tempo gasto em planejamento e recrutamento.

REFACS (online) 2014; 2(2):75-81. 


\section{MÉTODO}

Desenho: o Fit Extension foi projetado por e para entrega através do VCE, que é uma estrutura organizacional associada com a Universidade Virginia Tech., uma universidade de outorga pública (tipo de universidade americana com Sistema Cooperativo de Extensão) ${ }^{8}$.

O VCE tem um histórico de provimento de programas de educação nutricional e fornece um sistema de difusão para educadores em saúde que têm o potencial de atingir uma grande proporção da população em todo o estado ${ }^{6}$. Como parte de um estudo maior, este estudo representa o acompanhamento da execução do Fit Extension por 16 educadores em saúde do VCE, cada um em uma região diferente do estado da Virginia, EUA.

Cada educador em saúde foi contatado semanalmente durante quatro meses antes da data de início do programa (Abril de 2010) e a cada uma das oito semanas de execução do programa. Este artigo relata apenas os dados associados com a execução real do programa. Este estudo foi aprovado pelo Comitê de Ética da Universidade Virginia Tech., EUA.

Amostragem: dezoito educadores em saúde foram treinados para o Fit Extension e 16 executaram o programa. Todos os 16 que executaram o programa também participaram da avaliação de implementação do programa e eram do sexo feminino (idade média $=42,1 \pm 12,1$ anos). Os educadores serviam, em média, três e meio $\left(\mathrm{SD}_{ \pm} 1,45\right)$ municípios, cada um, e trabalhavam para o VCE por 7,85 anos (DP $\pm 9,69$ anos). Os participantes relataram gastar cerca da metade de suas horas de trabalho $(48,4 \%+$ 28,5\%) com nutrição e promoção da saúde.

Medidas: quando o Fit Extension foi apresentado aos educadores em saúde cada um recebeu uma planilha de monitoramento para acompanhamento das atividades e o tempo associados com o planejamento, treinamento e execução do programa. A cada semana os educadores em saúde foram contatados por e-mail ou telefone para

Figura 1. Percentagens Semanais de Relatórios de Quilometragem e fornecimento de feedbacks. Estado da Virginia, EUA. 2010. completar um questionário simples. Eles foram questionados se promoveram o Fit Extension como um programa baseado em equipes, no início do programa; e, em seguida, a cada semana o número de equipes que eles recolheram os relatórios de atividades físicas, se enviava mensagens personalizadas de acompanhamento e um boletim semanal. Eles também relataram atividades e tempo associados à preparação dos pacotes do programa para equipes participantes, inserindo informações em planilhas de monitoramento, recolhendo relatórios semanais de atividade física das equipes, desenvolvendo materiais locais para boletins informativos, comunicando-se com os participantes, conduzindo atividades para o programa e mantendo contato com os lideres das equipes.

Análise de Dados: utilizou-se estatística descritiva simples. Percentagens foram calculadas para determinar a proporção de educadores em saúde que completaram as tarefas de entrega. Foram calculadas as médias para o tempo gasto em diferentes aspectos da execução do programa.

\section{RESULTADOS}

Fidelidade da Execução do Programa

Todos os educadores em saúde $(n=16)$ executaram o programa utilizando equipes como foco central. Ao longo das oito semanas, os educadores reuniram relatórios de quilometragem semanal de líderes em média com $80,1 \%$ do tempo ( $S D_{ \pm} 0,08 \%$ ). 0 percentual de equipes que relatou sua quilometragem não variou significativamente por semana $(p>0,05)$. Os educadores em saúde forneceram feedback personalizado para as equipes em $72,5 \%$ (SD+.16\%) das vezes.

A proporção de feedback fornecido por educadores em saúde variou de forma significativa $(p<0,05)$ nas últimas semanas do programa (seis a oito semanas), sendo que a semana número três $(87,5 \%)$ teve proporção maior e as semanas sete e oito as mais baixas $(53,3 \%)$. 


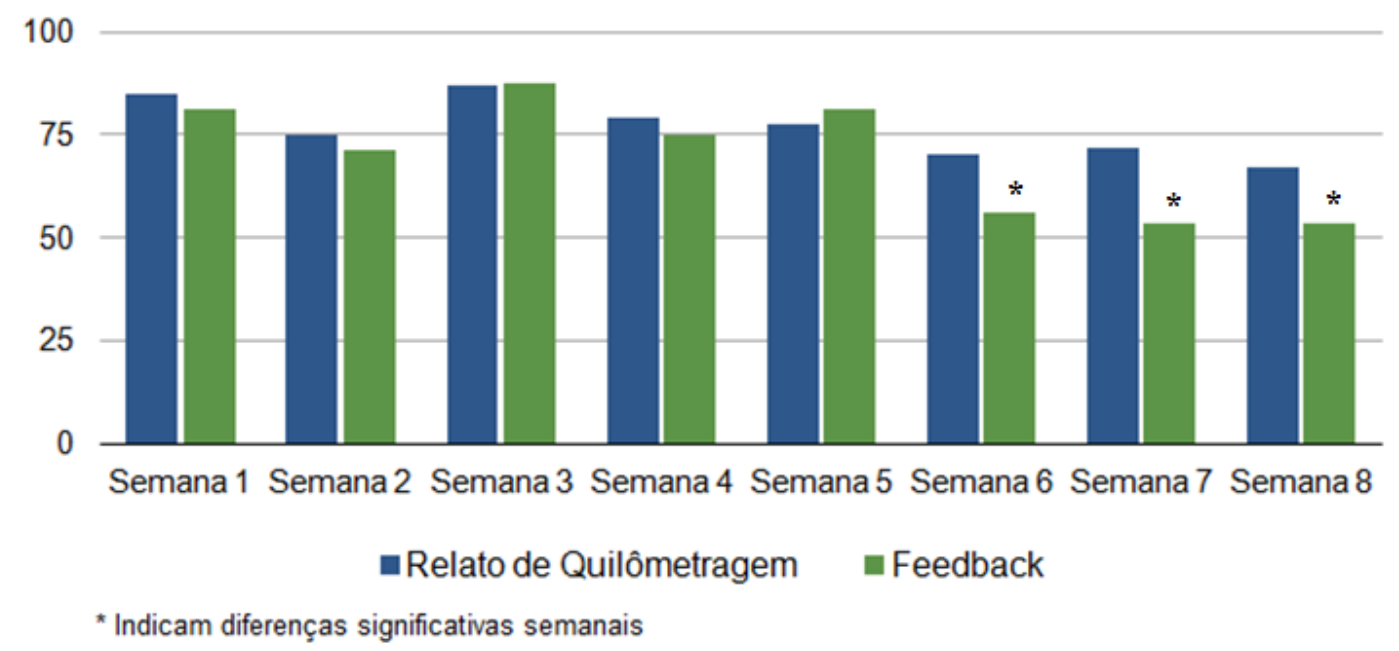

Tempo requerido para os Componentes do Programa

O instrumento de coleta capturou todos os componentes de execução do programa. Em média, os educadores em saúde trabalharam 10,75 horas por semana ( $S D \pm 16,27$ horas) em componentes relacionados à execução do programa.

A amplitude foi grande sendo que o mínimo de horas gastas na execução do programa foram 11 e o máximo foi de um educador em saúde que relatou ter gasto 241 horas totais com o programa. Como pode ser visto na Figura 2, os educadores passaram mais tempo na coleta da quilometragem das equipes (12,4 $\mathrm{SD} \pm 18,8$ horas); inserindo informações dos participantes em planilhas

Figura 2. Proporção do Total de Horas Gastas nos Componentes do Programa. Estado da Virginia, EUA. 2010.

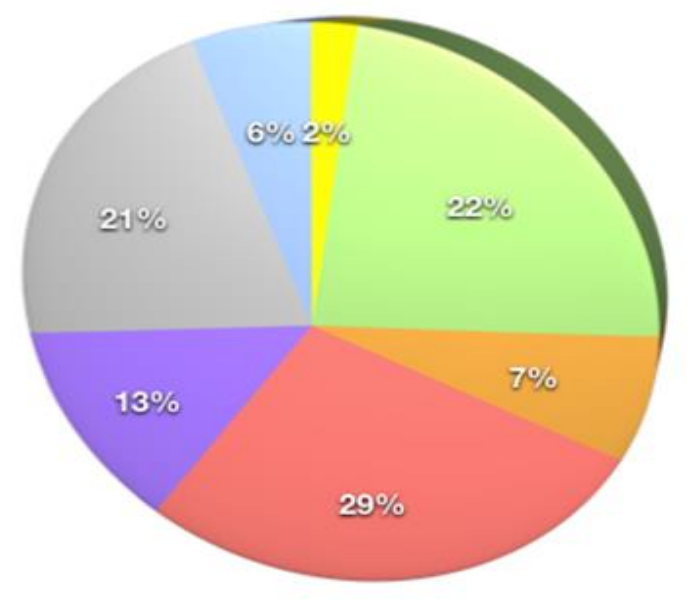

\section{DISCUSSÃO}

Em média, a implementação foi bastante elevada, mas diminuiu ao longo do curso do de acompanhamento $(9,47 \mathrm{SD} \pm 19,8$ horas $)$; comunicando-se com os participantes $(9,32$ $\mathrm{SD} \pm 13,7$ horas) e desenvolvendo materiais locais para boletins informativos $(5,7$ $\mathrm{SD} \pm 3,79$ horas).

Os componentes do programa em que os educadores em saúde gastaram a menor quantidade de tempo foram: preparando os pacotes do programa para equipes participantes $\quad(0,909 \quad \mathrm{SD} \pm 2,7 \quad$ horas $)$; conduzindo atividades relacionadas ao programa $(2,54 \mathrm{SD} \pm 7,17$ horas $)$ e mantendo contato com os lideres das equipes $(3,09$ $\mathrm{SD} \pm 2,81$ horas). A Figura 2 ilustra a quantidade média de horas gastas em cada componente de programa. 
equipe aos educadores em saúde, mas a proporção de agentes que ainda estavam fornecendo feedback diminuiu. Esta constatação está alinhada com outra pesquisa que mostra que mesmo ao nível do agente de execução, a adesão ao protocolo deve ser monitorada ${ }^{9}$. Também é sugerido que as estruturas que induzem à sustentabilidade da implementação (como incentivos para educadores em saúde) sejam necessárias para alcançar uma fidelidade contínua ${ }^{10}$.

Embora seja difícil encontrar outros dados sobre o tempo de execução na literatura sobre intervenções de base comunitária de atividade física e consumo de frutas e vegetais, verifica-se que o tempo necessário de comprometimento para com o Fit Extension, em todos os componentes de entrega do programa, foi relativamente baixo.

A média de tempo gasto nessas atividades foi menos de um dia de oito horas por semana, o que parece razoável neste contexto, já que os educadores informaram terem passado cerca de 20 horas por semana executando estratégias de nutrição e promoção da saúde. Portanto, um educador em saúde do VCE poderia executar o Fit Extension e ainda teria cerca de 12 horas por semana para completar as outras atividades de promoção da saúde, bem como manter-se dentro do intervalo de tempo que eles normalmente gastam nessas atividades.

Embora se encontrou um nível elevado de fidelidade da implementação, também nota-se que com o passar do tempo os educadores foram menos propensos a aderir a todos os componentes do programa. Enquanto não se sabe se essa redução na fidelidade ao final do programa foi relacionada com os requisitos do programa em si (como a exaustão da realização do programa) ou talvez demandas de trabalho concorrentes. É possível que o declínio no feedback fornecido para os participantes do programa poderia ter impactado o engajamento dos participantes e sucesso no programa. Pesquisas futuras poderiam investigar essas questões.
Igualmente, estas questões também têm implicações importantes para a avaliação de programas comunitários, particularmente aqueles executados ao longo de várias sessões. Em primeiro lugar, a efetividade desses programas pode ser afetada pelo grau de fidelidade da implementação.

Uma possível questão a ser investigada é se a efetividade do programa foi impactada pelo nível de fidelidade da implementação, tal como é necessário explorar a relação entre o grau em que os componentes do programa foram recebidos e seu efeito sobre o nível de sucesso dos participantes.

Em segundo lugar, a fidelidade da implementação poderia impactar a adoção de futuros programas, fornecendo uma avaliação precisa do tempo e os recursos necessários para execução dos mesmos.

Terceiro, a futura manutenção de comportamentos de saúde pelos participantes também pode ser afetada pelo grau em que "recebeu" o programa completo ou apenas partes do programa. Destaca-se que a fidelidade da implementação poderia impactar a sustentabilidade e fornecimento contínuo de programas futuros.

\section{CONCLUSÃO}

O objetivo geral deste trabalho foi fornecer um exemplo da avaliação de implementação de um programa de promoção da saúde de base comunitária. Foi documentado que o grau em que os componentes do programa foram executados variou como previsto, sendo que todos os educadores em saúde utilizaram a estrutura de dinâmica de grupo básica, mas nem todos trabalharam de maneira consistente com as equipes para coletar os relatórios semanais ou forneceram o feedback necessário.

Apesar do âmbito do presente estudo ser limitado à comunicação de tempo e atividades de execução da intervenção, de modo geral, os resultados sugerem a importância de incorporar as diferentes dimensões do modelo RE-AIM como parte do processo de planejamento e avaliação. As potenciais relações entre a fidelidade da implementação e outras dimensões do RE- 
AIM servem para destacar a importância de se abordar uma multiplicidade de fatores no planejamento e avaliação de programas de comportamento de saúde, a fim de aumentar o seu impacto na saúde coletiva e na sustentabilidade em longo prazo.

Por sua vez, a maioria destes fatores de validade externa (ou seja, alcance, adoção, implementação e manutenção) continua subrelatados.

Pesquisas futuras deveriam apresentar relatos sobre cada uma dessas dimensões e analisar o impacto e a relação entre esses fatores.

\section{REFERÊNCIAS}

1. Glasgow RE, Vogt TM, Boles SM. Evaluating the public health impact of health promotion interventions: The RE-AIM framework. Am J Public Health. 1999; 89:1322-7.

2. Cohen DJ et al. Fidelity Versus Flexibility Translating Evidence- Based Research into Practice. Am J Prev Med.2008; 35(5S).

3. Estabrooks PA et al. Move More: Translating Efficacious Physical Activity Intervention Principles into Effective Clinical Practice. International Journal of Sport and Exercise Psychology. 2011; 9:4-18.

4. Glasgow RE et al. Evaluating the impact of health promotion programs: using the RE-AIM framework to form summary measures for decision making involving complex issues. Health Educ Res. 2006; 21(5):688-94.

5. Estabrooks PA, Glasgow RE. Translating effective clinic-based physical activity interventions into practice. Am J Prev Med. 2006; 31(4 Suppl):S45-56.

6. Estabrooks PA et al. Determining the impact of Walk Kansas: applying a team-building approach to community physical activity promotion. Ann Behav Med. 2008; 36(1):1-12.

7. Carron Albert V, Spink KS. Team Building in an Exercise Setting. Sport Psychology. 2003; 7:8-18.

8. United States Department of Agriculture. National Institute of Food and Agriculture, 2013. Disponível em: http://www.csrees.usda.gov/Extension/ Acesso: 31/01/2014.
9. Bellig AJ et al. Enhancing treatment fidelity in health behavior change studies: best practices and recommendations from the NIH Behavior Change Consortium. Health Pscyhol. 2004; 23(5):443-51.

10. Casey MM et al. Sustaining health promotion programs within sport and recreation organizations. J Sci Med Sport. 2009; 12:113-8.

11. Glasgow RE, Linnan LA. Evaluation of theory-based interventions. In: Glanz K, Rimer BK, Viswanath K, eds. Health Behavior and Health Education: Theory, Research, and Practice. 4th ed. San Francisco, CA: JosseyBass, 487-508, 2008.

12. Allen $\mathrm{KC}$ et al. Understanding the internal and external validity of health literacy interventions: A systematic literature review using the RE-AIM framework. J Health Commun. 2011; 16(Suppl 3):55-72.

\section{CONTRIBUIÇÕES}

Samantha Marie Harden, Fábio Araújo Almeida, Wendy You e Paul Andrews Estabrooks tiveram iguais participações no desenho, supervisão das atividades de campo, análise de dados e redação do artigo.

\section{AGRADECIMENTOS}

Agradecimentos ao Instituto Nacional sobre Envelhecimento, EE.UU. para o financiamento do estudo (Grant\# s 025 667020118 AG AG 012113 AG 07907). 